\title{
Non-aneurysmal subarachnoid hemorrhage in a case of moyamoya disease
}

\author{
Mai A Mostafa* \\ El Demerdash Hospital, Ain Shams University, El-Mohamady, Al Waili, Egypt
}

\begin{abstract}
Moyamoya disease is bilateral progressive steno-occlusion of the supra-clinoid portions of internal carotid arteries subsequently formation of collateral vessels. Usually we see Moyamoya in children, but also frequently seen in adults, especially in the third or fourth decade of life. Non aneurysmal subarachnoid hemorrhage is very rare clinical manifestation of Moyamoya disease. This is an extremely rare condition and to our knowledge only five other cases have been described in the literature.
\end{abstract}

\section{Case report}

34-year old female patient presented with sudden headache and disturbed conscious level. She did CT brain which reveals diffuse subarachnoid hemorrhage (Figure 1). The clinician suspected rupture cerebral aneurysm and ordered CT cerebral angiography [1].

\section{Image findings}

CT brain which reveals bilateral diffuse subarachnoid hemorrhage and dilated ventricles (Figure 1). CT cerebral angiography reveals no aneurysms associated with diffuse narrowing of supraclinoid portions of both internal carotid arteries (Figure 2). Diagnostic angiography of right internal carotid artery reveals diffuse narrowing of the supraclinoid portion (Figure 3). Diagnostic angiography of left internal carotid artery reveals diffuse narrowing of the supra-clinoid portion with multiple collaterals (Figure 4). Diagnostic angiography reveals multiple meningeal collaterals (Figure 5).

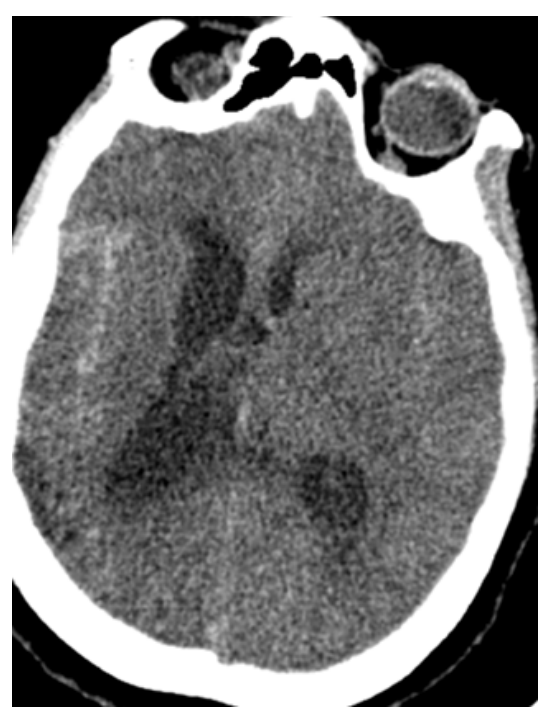

Figure 1. axial CT cut of 30 years old female patient presented with sever headache, the CT reveals subarchnoid hemorrhage

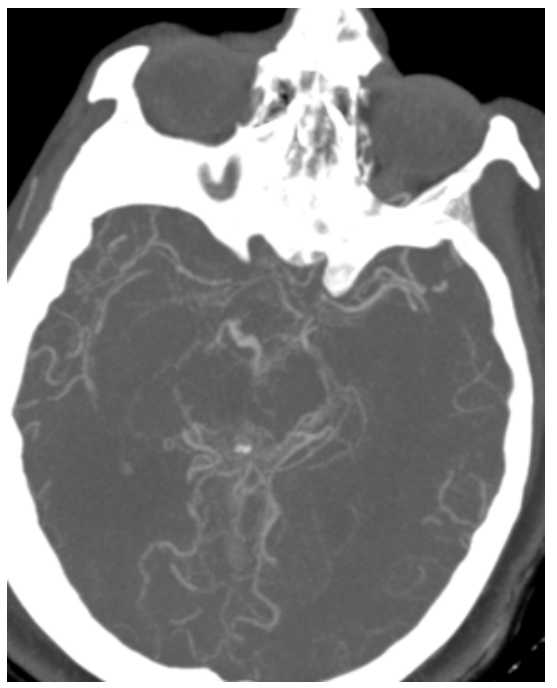

Figure 2. Axial cut of CTA reveals no aneurysms associated with diffuse narrowing of supraclinoid portions of both internal carotid arteries associated with basal collaterals

\section{Management of moyamoya}

Conservative and surgical treatment: Causal treatment of the disease is not known. Mild Cases are treated conservatively. To avoid ischemia and strokes, Medical treatment could be used and it is include antiplatelet and anticoagulative therapy which rarely used, due to the risk of bleeding. In severe cases, surgery is indicated which includes direct anastomoses, indirect procedures, and combined therapies. Anastomoses are usually performed between the superficial temporal artery and the middle cerebral artery. Indirect procedures include synangiosis involving placement of vascularised tissue in the brain cortex, in order to promote neoangiogenesis. Recently, there has also

*Correspondence to: Mai A Mostafa, El Demerdash Hospital, Ain Shams University, El-Mohamady, Al Waili, Egypt, E-mail: mayson.1988@yahoo.com

Received: August 23, 2019; Accepted: September 12, 2019; Published: September 16, 2019 


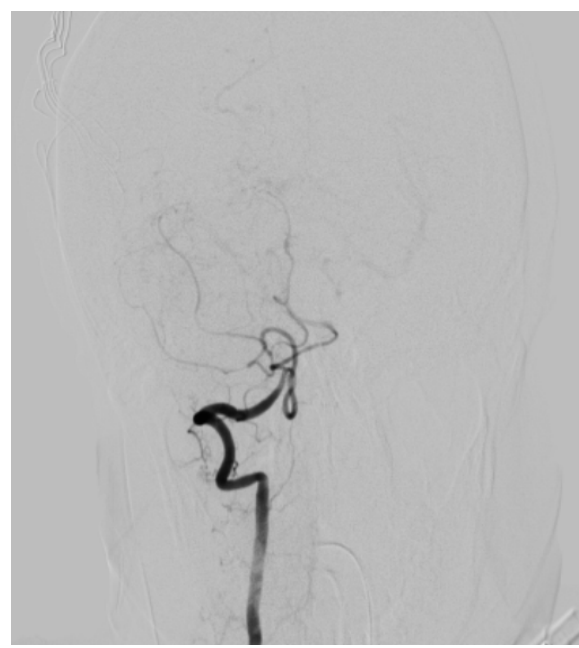

Figure 3. AP view of diagnostic angiography of right internal carotid artery reveals diffuse narrowing of the supra-clinoid portion

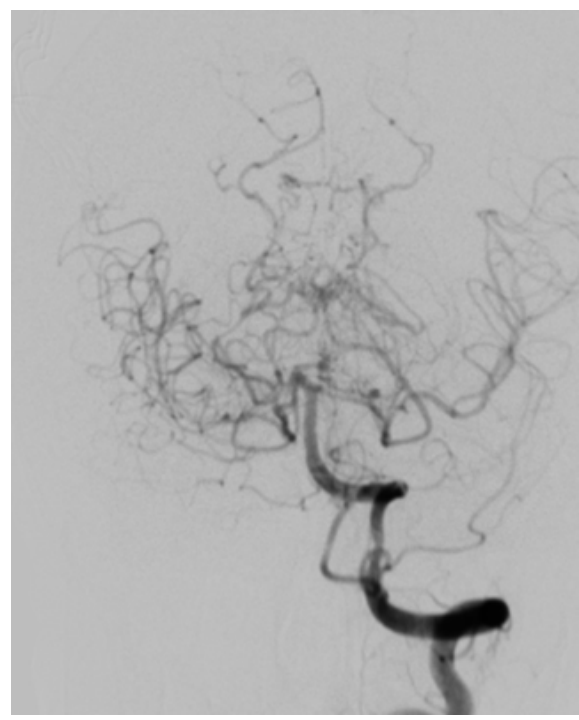

Figure 4. AP view of diagnostic angiography of left internal carotid artery reveals diffuse narrowing of the supra-clinoid portion with multiple collaterals

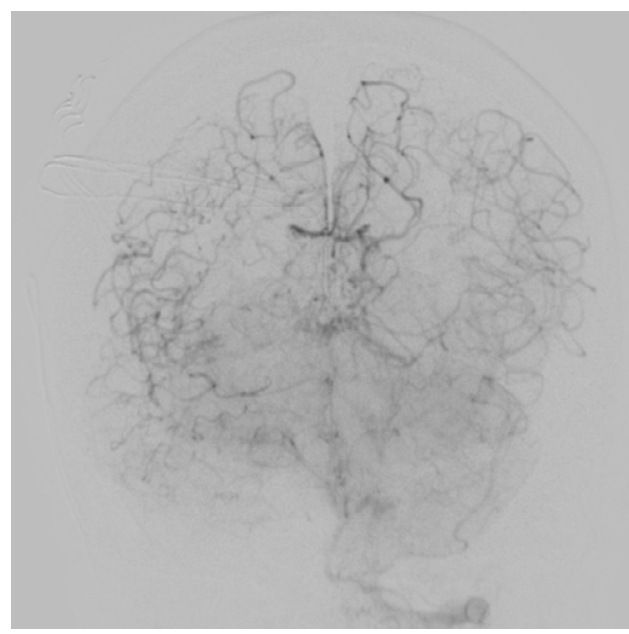

Figure 5. AP view of diagnostic angiography reveals multiple meningeal collaterals been reported a successful implantation of a stent to the internal carotid artery in a young woman with moyamoya syndrome and recurrent TIA symptoms [2].

\section{Japan adult moyamoya (JAM) trial and guideline recommendation}

The most recent guideline for moyamoya in Japan recommends direct revascularization surgery for the patients with MMD manifesting as cerebral ischemic symptoms. Regarding hemorrhagic-onset patients, there had been a controversy whether surgical revascularization has potential role for reducing the risk of re-bleeding [3].

\section{Discussion}

Clinical presentation in adults include ischemic stroke and recurrent hemorrhages. Rebleeding carry high morbidity and mortality [4].

\section{Changes of the disease:}

Stenosis of the terminal parts of internal carotid arteries and/ or proximal parts of middle and anterior cerebral arteries.

Different degree of stenosis and occlusions of large arteries of the anterior part of the Willis circle.

Development of the collateral vasculature that produces a typical angiographic image, called 'clouds of smoke' or 'puff of cigarette smoke' [3].

The collateral circulation are formed of small or medium vessels as a result of widening of the existing vessels or development of new perforating arteries . they are branching from intracranial parts of internal carotid arteries, posterior cerebral arteries or anterior choroidal arteries. The collaterals anastomose the distal branches of the middle cerebral arteries. There are three main pathways of collateral circulation - parenchymal, meningeal, and transdural [3]. Moyamoya disease may associated with aneurysmal subarchnoid and intracranial hemorrhage [5]. In our case this was a primary subarchnoid hemorrhage likely secondary to rupture collaterals.

\section{Imaging}

CT shows the ischemic or hemorrhagic changes. Cerebral angiography plays a crucial role in the assessment of moyamoya disease. The characteristic angiographic findings are traditionally described in stages, as the disease progresses toward total ICA occlusion and complete cerebral perfusion through external carotid and vertebral collateralizations [6]. The six-stage classification of Suzuki is used to grade moyamoya disease.

\section{Narrowing of carotid arteries}

2. Initial appearance of moyamoya vessels

3. Intensification of moyamoya vessels

4. Minimization of moyamoya vessels

5. Reduction of moyamoya vessels

6. Disappearance of moyamoya vessels

Magnetic resonance imaging (MRI) and magnetic resonance angiography (MRA) is useful noninvasive technique that diagnose the steno-occlusive changes of carotid vessels [6].

\section{Learning points}

Primary subarachnoid hemorrhage could occur in moyamoya disease without aneurysms. 


\section{References}

1. Toscano M, Puledda F, Vigano A, Vicenzini E, Guidetti G, et al. (2014) Hemodynamic features of non-aneurysmal subarachnoid hemorrhage in a case of familial Moyamoya disease: A transcranial doppler ultrasound study. Eur Neurol 72: 330-332. [Crossref]

2. Tarasow E, Kułakowska A, Lukasiewicz A, Kapica-Topczewska K, KornelukSadzynska A, et al. (2011) Moyamoya disease: Diagnostic imaging. Pol J Radiol 76: 73-79. [Crossref]
3. Fujimura M, Tominaga T (2019) Hemorrhagic Moyamoya Disease: A recent update. $J$ Korean Neurosurg Soc 62: 136-143. [Crossref]

4. Kang S, Liu X, Zhang D, Wang R, Zhang Y, et al. (2019) Natural course of Moyamoya disease in patients with prior hemorrhagic stroke. Stroke 50: 1060-1066. [Crossref]

5. Aoki N, Mizutani H (1984) Does moyamoya disease cause subarachnoid hemorrhage? Review of 54 cases with intracranial hemorrhage confirmed by computerized tomography. J Neurosurg 60: 348-353. [Crossref]

6. Zipfel GJ, Fox DJ, Rivet DJ (2005) Moyamoya disease in adults: The role of cerebral revascularization. Skull Base 15: 27-41. [Crossref]

Copyright: ( 2019 Mostafa MA. This is an open-access article distributed under the terms of the Creative Commons Attribution License, which permits unrestricted use, distribution, and reproduction in any medium, provided the original author and source are credited. 